\title{
Galhas induzidas por artrópodes em Floresta Estacional Semidecidual no Sul do Brasil
}

\author{
Fernando Albuquerque $\operatorname{Luz}^{1 *}$ \\ Ana Paula Moraes Goetz ${ }^{2}$ \\ ${ }^{1}$ Universidade Federal do Amazonas, Instituto de Saúde e Biotecnologia \\ Estrada Coari/Mamiá, 305, CEP 69.460-000, Coari - AM, Brasil \\ ${ }^{2}$ Universidade Federal do Rio Grande do Sul, PPG - Biologia Animal, Instituto de Biociências \\ Avenida Bento Gonçalves, 9500, Setor 4, CEP 91.501-970, Porto Alegre - RS, Brasil \\ * Autor para correspondência \\ fernandoaluz@gmail.com
}

Submetido em 10/11/2020

Aceito para publicação em 02/03/2021

\section{Resumo}

Neste estudo descrevemos a riqueza de morfotipos de galhas induzidas por artrópodes em uma área de Floresta Estacional Semidecidual no Rio Grande do Sul, Brasil. Nós encontramos 71 espécies de artrópodes galhadores, a maioria delas Cecidomyiidae (Diptera) associada a 51 espécies de plantas hospedeiras de 27 famílias. Asteraceae foi a família hospedeira mais representativa (18 morfotipos). A maior parte das galhas foi induzida em ramos (56\%) e a forma mais comum foi fusiforme (43,6\%). Dezenove novos morfotipos de galhas foram registrados para o Rio Grande do Sul e para o Brasil, mostrando a importância desse tipo de estudo para galhadores em regiões pouco exploradas, como o Sul do Brasil.

Palavras-Chave: Cecidomyiidae; Indutores de galha; Planta hospedeira; Rio Grande do Sul; Riqueza de galhas

\section{Abstract}

Gall-inducing arthropods of semideciduous forest in southern Brazil. In this study, we describe the richness of galls induced by arthropods in an area of the Semideciduous Seasonal Forest in Rio Grande do Sul State, Brazil. We found 71 species of galling arthropod, most of them Cecidomyiidae (Diptera), associated with 51 host plants species from 27 families. Asteraceae was the most representative host family (18 morphotypes). Most galls were induced in stems (56\%) and the most common shape was fusiform (43.6\%). Nineteen new gall morphotypes were registered for Rio Grande do Sul and Brazil, showing the importance of this type of study for gallers in underexplored regions, such as Southern Brazil.

Key words: Cecidomyiidae; Gall inducer; Gall richness; Host plant; Rio Grande do Sul 


\section{Introdução}

Galhas são estruturas formadas pelo crescimento anormal de tecidos vegetais em resposta a estímulos causados por insetos, ácaros e outros organismos (MANI, 1964; RAMAN, 2007). Essa estrutura é considerada o fenótipo estendido de seu indutor (STONE; SCHÖNROGGE, 2003), já que cada espécie galhadora é responsável por induzir um morfotipo único, ou seja, uma galha com fisiologia e anatomia distintas das induzidas por outras espécies (SHORTHOUSE et al., 2005). Grande parte dos galhadores é hospedeiro específico, principalmente Cecidomyiidae (Diptera) (CARNEIRO et al., 2009), portanto, morfotipos de galhas são frequentemente utilizados em inventários como substitutos para o registro das espécies de artrópodes que as induzem.

O Brasil é o país que apresenta o maior número de publicações sobre artrópodes galhadores na América Latina (GRANDEZ-RIOS et al., 2015). Entretanto, nem todas as regiões e biomas do país foram explorados com a mesma intensidade. Grande parte destas publicações foram realizadas no Sudeste do país, enquanto as demais regiões, principalmente Norte e Sul, permanecem pouco exploradas (ARAÚJO, 2018; ARAÚJO et al., 2019).

A Mata Atlântica é um dos principais biomas brasileiros e o mais investigado até o momento em relação à diversidade de artrópodes galhadores no Brasil (ARAÚJO et al., 2019). Este domínio é composto por diferentes tipos de formações florestais e de ecossistemas associados (e.g. manguezais e restingas). Dentro destes tipos de formações florestais, os estudos foram realizados predominantemente em áreas de restinga (e.g. MAIA, 2001; 2013a; MAIA et al., 2008; BREGONCI et al., 2010; MAIA; OLIVEIRA, 2010) e de Floresta Ombrófila Densa (MAIA et al., 2014; MAIA; MASCARENHAS, 2017; MAIA; SIQUEIRA, 2020) na região Sudeste do país. Outras fitofisionomias da Mata Atlântica permanecem pouco exploradas, como a Floresta Estacional Semidecidual. Inventários de insetos galhadores para esse tipo de formação florestal estão localizados em maior número na região Sudeste (FLOR et al., 2018; RIBEIRO et al., 2019; COSTA; ARAÚJO, 2019), enquanto a fauna de outras regiões do país é pouco conhecida. Esse é o caso da região Sul, que registra apenas dois inventários de galhadores em áreas de Floresta Estacional Semidecidual (SANTOS; RIBEIRO, 2015; GOETZ et al., 2018).

Nesse contexto, nosso objetivo é contribuir para o conhecimento dos artrópodes galhadores e suas plantas hospedeiras da região Sul do Brasil, investigando uma área de Floresta Estacional Semidecidual no Rio Grande do Sul.

\section{Material e Métodos}

A área de estudo foi uma propriedade particular (3135'37.4”S 52³4'52.6”W) que apresenta áreas de mata e de campo ao longo dos 50 ha do local. A área está situada no município de Morro Redondo, Rio Grande do Sul, Brasil. A altitude do município é de 245 $\mathrm{m}$ e o clima da região é do tipo Cfa (classificação de Köppen) com temperaturas médias anuais entre $13,8^{\circ} \mathrm{C}$ e $22,9^{\circ} \mathrm{C}$ (MORENO, 1961). A área onde foi realizado o estudo pertence à Floresta Estacional Semidecidual (TEIXEIRA et al., 1986), esse tipo de formação vegetal é caracterizado, pela predominância de florestas que possuem a característica de perda das folhas durante o inverno, atingindo entre 25 e $50 \%$ de suas espécies arbóreas (IBGE, 2012).

Foram realizadas 12 amostragens mensais entre junho de 2014 e maio de 2015. Em cada expedição, sete trilhas pré-estabelecidas com $1 \mathrm{~km}$ de comprimento cada (essas trilhas abrangeram também pequenas áreas de campo entre um fragmento de mata e outro, assim como a mata ciliar) foram percorridas à procura de galhas. Entre cada trilha, foi estabelecido $1 \mathrm{~km}$ de distância entre as extremidades mais próximas. Foi realizada uma busca ativa, sem cronometragem de tempo, em um raio de $1 \mathrm{~km}$, marcado por GPS (Garmin Etrex) nas trilhas pré-estabelecidas. Um único coletor realizou todas as coletas e este não se distanciava mais de $10 \mathrm{~m}$ para nenhum lado da trilha em busca de galhas. As sete trilhas percorridas eram amostradas sempre em no máximo três dias corridos.

As galhas avistadas em campo foram fotografadas, coletadas e, em laboratório, separadas por morfotipo, isto é, classificadas com base em sua morfologia 
externa em combinação com órgão galhado e espécie de planta hospedeira (CARNEIRO et al., 2009). Parte das galhas coletadas foi dissecada sob microscópio estereoscópico para obtenção do inseto indutor imaturo, caracterização e descrição da galha (forma, coloração e número de câmaras) (ISAIAS et al., 2013). A outra parte foi acondicionada em sacos plásticos para a obtenção dos artrópodes galhadores adultos que, após a sua emergência, foram armazenados em álcool $70 \%$ e identificados até o menor nível taxonômico possível (com base na especificidade parasita-hospedeiro). As plantas hospedeiras não identificadas em campo tiveram alguns dos seus ramos coletados para identificação através de especialistas. A lista de táxons das plantas hospedeiras foi organizada de acordo com o sistema de classificação do APG IV (2016).

\section{Resultados}

Foram registrados 71 morfotipos de galhas para seis ordens de artrópodes (Tabela 1; Figuras 1 a 4). A maioria das galhas foi induzida por Cecidomyiidae (Diptera), representando 56\% $(\mathrm{n}=41)$ das espécies registradas no estudo. Lepidoptera registrou apenas $11 \%(\mathrm{n}=8)$, seguida por Coleoptera $(8 \%, \mathrm{n}=6)$, Hemiptera $(7 \%, \mathrm{n}=$ $5)$, Diptera $(6 \%, n=4)$, Acari $(5 \%, n=3)$, Hymenoptera $(2 \%, n=1)$ e em $5 \%(n=3)$ os indutores não foram identificados. Entre os morfotipos coletados, 18 (24\%) representam novos registros, comparando com literatura pertinente, ou seja, nenhuma menção a uma galha com anatomia externa semelhante em uma mesma hospedeira das aqui listadas pode ser encontrada. Apenas $10(15 \%)$ entre o total de morfotipos registrados são induzidos por espécies já descritas.

TABELA 1: Plantas hospedeiras, características morfológicas e indutores das galhas amostradas na Floresta Estacional Semidecidual no Rio Grande do Sul, Brasil. Número de câmaras na galha: M, monotálamo; P, politálamo. * Indica novos registros, baseados na morfologia da galha e na espécie de planta, segundo literatura disponível.

\begin{tabular}{|c|c|c|c|c|c|}
\hline Planta Hospedeira & Órgão & Forma & Cor & Indutor & Figura \\
\hline \multicolumn{6}{|l|}{ Anacardiaceae } \\
\hline Lithraea brasiliensis Marchand & Folha & Lenticular $^{\mathrm{M}}$ & Verde & Hemiptera & $1 \mathrm{~A}^{*}$ \\
\hline Schinnus polygamus Cabrera & Ramo & Globoide $^{\mathrm{M}}$ & Verde & $\begin{array}{c}\text { Cecidoses eremita Curtis, } 1835 \\
\text { (Lepidoptera) }\end{array}$ & $1 \mathrm{~B}$ \\
\hline Schinnus polygamus Cabrera & Ramo & Globoide $^{\mathrm{M}}$ & Marrom & $\begin{array}{c}\text { Eucecidoses minutanus Brèthes, } 1916 \\
\text { (Lepidoptera) }\end{array}$ & $1 \mathrm{C}$ \\
\hline Schinnus polygamus Cabrera & Ramo & Fusiforme $^{\mathrm{M}}$ & Marrom & $\begin{array}{c}\text { Oliera argentinana } \text { Brèthes, } 1916 \\
\text { (Lepidoptera) }\end{array}$ & $1 \mathrm{D}$ \\
\hline Schinnus polygamus Cabrera & Folha & Lenticular ${ }^{\mathrm{M}}$ & $\begin{array}{c}\text { Verde/ } \\
\text { Vermelha }\end{array}$ & $\begin{array}{c}\text { Calophya duvauae (Scott, 1882) } \\
\text { (Hemiptera) }\end{array}$ & $1 \mathrm{E}$ \\
\hline \multicolumn{6}{|l|}{ Asteraceae } \\
\hline Baccharis articulata (Lam.), Pers & Ramo & Bivalve $^{\mathrm{M}}$ & Verde & Cecidomyiidae, Diptera & $1 \mathrm{~F}^{*}$ \\
\hline Baccharis dracunculifolia DC & Folha & Fusiforme $^{\mathrm{M}}$ & Verde & $\begin{array}{c}\text { Baccharopelma dracunculifoliae } \\
\text { Burckhardt, Espírito-Santo, Fernandes \& } \\
\text { Malenovský, } 2004 \text { (Hemiptera) }\end{array}$ & $1 \mathrm{G}$ \\
\hline Baccharis dracunculifolia DC & Gema & Roseta $^{\mathrm{M}}$ & Verde & Cecidomyiidae (Diptera) & $1 \mathrm{H}$ \\
\hline Baccharis dracunculifolia DC & Gema & Roseta $^{\mathrm{M}}$ & Verde & Cecidomyiidae (Diptera) & 1I \\
\hline Baccharis dracunculifolia DC & Ramo & Fusiforme $^{\mathrm{M}}$ & Verde & Lepidoptera & $1 \mathrm{~J}$ \\
\hline Baccharis punctulata DC & Folha & Lenticular $^{\mathrm{M}}$ & Marrom & Cecidomyiidae (Diptera) & $1 \mathrm{~K}^{*}$ \\
\hline Baccharis tridentata Vahl & Ramo & Amorfa $^{\mathrm{M}}$ & Verde & Cecidomyiidae (Diptera) & $1 \mathrm{~L}^{*}$ \\
\hline Baccharis trimera (Less) DC & Ramo & Globoide $^{\mathrm{M}}$ & Verde & Cecidomyiidae (Diptera) & $1 \mathrm{M}^{*}$ \\
\hline Baccharis trimera (Less) DC & Ramo & Fusiforme $^{\mathrm{M}}$ & Verde & Cecidomyiidae (Diptera) & $1 \mathrm{~N}^{*}$ \\
\hline $\begin{array}{l}\text { Dasyphyllum spinescens (Less) } \\
\text { Cabrera }\end{array}$ & $\begin{array}{c}\text { Folha } \\
\text { (pecíolo) }\end{array}$ & Fusiforme $^{\mathrm{M}}$ & Marrom & Cecidomyiidae (Diptera) & 10 \\
\hline Eupatorium inulifolium Kunth & Ramo & Globoide $^{\mathrm{P}}$ & Vermelha & Tephriitidae (Diptera) & $1 \mathrm{P}$ \\
\hline Mikania glomerata Spreng. & Gema & Globoide $^{\mathrm{P}}$ & Verde & $\begin{array}{c}\text { Perasphondylia mikaniae Gagné, } 2001 \\
\text { (Diptera) }\end{array}$ & $1 Q$ \\
\hline
\end{tabular}




\begin{tabular}{|c|c|c|c|c|c|}
\hline Planta Hospedeira & Órgão & Forma & Cor & Indutor & Figura \\
\hline Mikania hastato-cordata Malme & Ramo & Fusiforme $^{\mathrm{M}}$ & Vermelha & Cecidomyiidae (Diptera) & $1 \mathrm{R}^{*}$ \\
\hline Mikania hastato-cordata Malme & Folha & Amorfa $^{\mathrm{M}}$ & Verde & Acari & $1 \mathrm{~S}^{*}$ \\
\hline $\begin{array}{l}\text { Moquiniastrum polymorphum } \\
\text { (Less.) G. Sancho }\end{array}$ & Ramo & Fusiforme $^{\mathrm{M}}$ & Verde & Cecidomyiidae (Diptera) & $1 \mathrm{~T}$ \\
\hline $\begin{array}{l}\text { Senecio brasiliensis (Spreng.) } \\
\text { Less. }\end{array}$ & Ramo & Fusiforme $^{\mathrm{M}}$ & Verde & Coleoptera & $2 \mathrm{~A}^{*}$ \\
\hline Solidago chilensis Meyen & Ramo & Fusiforme $^{\mathrm{P}}$ & Verde & Tephritidae (Diptera) & $2 \mathrm{~B}^{*}$ \\
\hline Trixis praestans (Vell.) Cabrera & Ramo & Fusiforme $^{\mathrm{P}}$ & Verde & Diptera & $2 \mathrm{C}$ \\
\hline $\begin{array}{l}\text { Vernonanthura discolor } \\
\text { (Spreng.) H. Rob. }\end{array}$ & Ramo & Fusiforme $^{\mathrm{P}}$ & Verde & Diptera & $2 \mathrm{D}$ \\
\hline \multicolumn{6}{|l|}{ Bignoniaceae } \\
\hline $\begin{array}{l}\text { Pithecoctenium crucigerum (L.) } \\
\text { A. H. Gentry }\end{array}$ & Ramo & Fusiforme $^{\mathrm{M}}$ & Verde & Cecidomyiidae (Diptera) & $2 \mathrm{E}^{*}$ \\
\hline \multicolumn{6}{|l|}{ Cannabaceae } \\
\hline Celtis iguanea (Jacq.) Sarg. & $\begin{array}{c}\text { Folha } \\
\text { (nervura) }\end{array}$ & Fusiforme $^{\mathrm{M}}$ & Verde & Cecidomyiidae (Diptera) & $2 \mathrm{~F}$ \\
\hline \multicolumn{6}{|l|}{ Dioscoreaceae } \\
\hline Dioscorea multiflora Griseb. & $\begin{array}{c}\text { Folha } \\
\text { (pecíolo) }\end{array}$ & Globoide $^{\mathrm{M}}$ & Marrom & Cecidomyiidae (Diptera) & $2 \mathrm{G}$ \\
\hline \multicolumn{6}{|l|}{ Escalloniaceae } \\
\hline Escalloniabifida Link \& Otto & Ramo & Roseta $^{\mathrm{M}}$ & Verde & Coleoptera & $2 \mathrm{H}$ \\
\hline \multicolumn{6}{|l|}{ Euphorbiaceae } \\
\hline $\begin{array}{l}\text { Sapium glandulosum (L.) } \\
\text { Morong }\end{array}$ & Folha & Lenticular $^{\mathrm{M}}$ & Vermelha & Psyllidae (Hemiptera) & $2 \mathrm{I}$ \\
\hline $\begin{array}{l}\text { Sebastiania commersoniana } \\
\text { (Baill.) L.D.Sm. \&Downs }\end{array}$ & Folha & Lenticular $^{\mathrm{M}}$ & Vermelha & Cecidomyiidae (Diptera) & $2 \mathrm{~J}$ \\
\hline $\begin{array}{l}\text { Sebastiania commersoniana } \\
\text { (Baill.) L.D.Sm. \&Downs }\end{array}$ & $\begin{array}{c}\text { Folha } \\
\text { (nervura) }\end{array}$ & Fusiforme $^{\mathrm{M}}$ & Verde & Cecidomyiidae (Diptera) & $2 \mathrm{~K}$ \\
\hline $\begin{array}{l}\text { Sebastiania commersoniana } \\
\text { (Baill.) L.D.Sm. \&Downs }\end{array}$ & Folha & Lenticular $^{\mathrm{M}}$ & Verde & Cecidomyiidae (Diptera) & $2 \mathrm{~L}$ \\
\hline $\begin{array}{l}\text { Sebastiania commersoniana } \\
\text { (Baill.) L.D.Sm. \&Downs }\end{array}$ & Ramo & Globoide $^{\mathrm{M}}$ & Marrom & Não identificado & $2 \mathrm{M}$ \\
\hline \multicolumn{6}{|l|}{ Lamiaceae } \\
\hline Ocimum selloi Benth. & Ramo & Fusiforme $^{\mathrm{M}}$ & Vermelha & Cecidomyiidae (Diptera) & $2 \mathrm{~N}^{*}$ \\
\hline Salvia guaranítica St. - Hill. & Ramo & Fusiforme $^{\mathrm{M}}$ & Verde & Coleoptera & $2 \mathrm{O}^{*}$ \\
\hline \multicolumn{6}{|l|}{ Lythraceae } \\
\hline Heimia myrtifolia Cham. \&Schltd & Ramo & Fusiforme $^{\mathrm{M}}$ & Marrom & Lepidoptera & $2 \mathrm{P}$ \\
\hline \multicolumn{6}{|l|}{ Malvaceae } \\
\hline Luehea divaricata Mart. \&Zucc. & Ramo & Globoide $^{\mathrm{M}}$ & Marrom & Cecidomyiidae (Diptera) & $2 \mathrm{Q}$ \\
\hline Luehea divaricata Mart. \&Zucc. & Ramo & Fusiforme $^{\mathrm{M}}$ & Marrom & Não identificado & $2 \mathrm{R}$ \\
\hline Luehea divaricata Mart. \&Zucc. & Ramo & Globoide $^{\mathrm{M}}$ & Verde & Cecidomyiidae (Diptera) & $2 \mathrm{~S}$ \\
\hline Sida sp. & Ramo & Fusiforme $^{\mathrm{M}}$ & Verde & Coleoptera & $2 \mathrm{~T}$ \\
\hline \multicolumn{6}{|l|}{ Melastomataceae } \\
\hline $\begin{array}{l}\text { Miconia hiemalis A. St. - Hill } \\
\text { \& Naudin }\end{array}$ & Ramo & Fusiforme $^{\mathrm{M}}$ & Marrom & Lepidoptera & $3 A^{*}$ \\
\hline $\begin{array}{l}\text { Miconia pusilliflora (DC.) } \\
\text { Naudin }\end{array}$ & $\begin{array}{c}\text { Folha } \\
\text { (nervura) }\end{array}$ & Fusiforme $^{\mathrm{M}}$ & Verde & Cecidomyiidae (Diptera) & 3B \\
\hline \multicolumn{6}{|l|}{ Meliaceae } \\
\hline Trichilia catigua A. Juss. & Folha & Lenticular $^{\mathrm{M}}$ & Amarela & Hemiptera & $3 \mathrm{C}^{*}$ \\
\hline \multicolumn{6}{|l|}{ Myrtaceae } \\
\hline $\begin{array}{l}\text { Blepharocalyx salicifolius } \\
\text { (Kunth) O. Berg }\end{array}$ & Folha & Clavada $^{\mathrm{M}}$ & Verde & Acari & $3 \mathrm{D}$ \\
\hline
\end{tabular}




\begin{tabular}{|c|c|c|c|c|c|}
\hline Planta Hospedeira & Órgão & Forma & Cor & Indutor & Figura \\
\hline Eugenia uniflora $\mathrm{L}$. & Folha & Cônica ${ }^{\mathrm{M}}$ & $\begin{array}{l}\text { Vermelha/ } \\
\text { Verde }\end{array}$ & Clinodiplosis profusa Maia, 2001 (Diptera) & $3 \mathrm{E}$ \\
\hline Eugenia uniflora L. & Folha & Lenticular $^{\mathrm{M}}$ & Branco & Cecidomyiidae (Diptera) & $3 \mathrm{~F}$ \\
\hline Eugenia uniflora L. & Folha & Globoide $^{\mathrm{M}}$ & Verde & Cecidomyiidae (Diptera) & $3 \mathrm{G}$ \\
\hline Eugenia uniflora L. & Ramo & Fusiforme $^{\mathrm{M}}$ & Marrom & Hymenoptera & $3 \mathrm{H}$ \\
\hline $\begin{array}{l}\text { Myrcianthes gigantea (D. } \\
\text { Legrand) D. Legrand }\end{array}$ & Folha & Clavada $^{\mathrm{M}}$ & Branco & Cecidomyiidae (Diptera) & $3 \mathrm{I}$ \\
\hline Psidium cattleianum Sabine & Folha & Fusiforme $^{\mathrm{M}}$ & Branco & $\begin{array}{l}\text { Tectococcus ovatus Hempel, } 1900 \\
\text { (Hemiptera) }\end{array}$ & $3 \mathrm{~J}$ \\
\hline \multicolumn{6}{|l|}{ Nyctaginaceae } \\
\hline Guapira opposita (Vell.) Reiz. & Folha & Lenticular $^{\mathrm{M}}$ & Verde & $\begin{array}{c}\text { Bruggmannia elongata Maia \& Couri, } \\
1993 \text { (Diptera) }\end{array}$ & $3 \mathrm{~K}$ \\
\hline Guapira opposita (Vell.) Reiz. & Ramo & Roseta $^{P}$ & Verde & $\begin{array}{c}\text { Pisphondylia brasiliensis Couri \& Maia, } \\
1992 \text { (Diptera) }\end{array}$ & $3 \mathrm{~L}$ \\
\hline \multicolumn{6}{|l|}{ Piperaceae } \\
\hline Piper xylosteoides (Kunth.) Steud & Ramo & Globoide $^{\mathrm{M}}$ & Marrom & Cecidomyiidae (Diptera) & $3 \mathrm{M}^{*}$ \\
\hline \multicolumn{6}{|l|}{ Polygonaceae } \\
\hline Polygonum acuminatum Kunth. & Ramo & Fusiforme $^{\mathrm{P}}$ & Verde & Cecidomyiidae (Diptera) & $3 \mathrm{~N}^{*}$ \\
\hline \multicolumn{6}{|l|}{ Primulaceae } \\
\hline Myrsine coriacea (Sw.) R. Br. & Ramo & Fusiforme $^{\mathrm{M}}$ & Marrom & Coleoptera & $3 \mathrm{O}$ \\
\hline $\begin{array}{l}\text { Myrsine laetevirens (Mez) } \\
\text { Arechav. }\end{array}$ & Ramo & Fusiforme $^{\mathrm{M}}$ & Verde & Coleoptera & $3 \mathrm{P}$ \\
\hline Myrsine umbellata Mart. & Ramo & Fusiforme $^{\mathrm{M}}$ & Verde & Lepidoptera & $3 \mathrm{Q}$ \\
\hline \multicolumn{6}{|l|}{ Rhamnaceae } \\
\hline Scutia buxifolia Reissek. & Ramo & Globoide $^{\mathrm{P}}$ & Marrom & Cecidomyiidae (Diptera) & $3 \mathrm{R}$ \\
\hline Scutia buxifolia Reissek. & Ramo & Fusiforme $^{\mathrm{M}}$ & Marrom & Não identificado & $3 \mathrm{~S}$ \\
\hline \multicolumn{6}{|l|}{ Rubiaceae } \\
\hline Psychotria carthagenensis Jacq. & Folha & Lenticular $^{\mathrm{M}}$ & Verde & Cecidomyiidae (Diptera) & $3 \mathrm{~T}$ \\
\hline \multicolumn{6}{|l|}{ Salicaceae } \\
\hline Casearia sylvestris $\mathrm{Sw}$. & Folha & Lenticular $^{\mathrm{M}}$ & Verde & Cecidomyiidae (Diptera) & $4 \mathrm{~A}$ \\
\hline \multicolumn{6}{|l|}{ Sapindaceae } \\
\hline Allophylus edulis Radlk. & Ramo & Fusiforme $^{\mathrm{M}}$ & Verde & Lepidoptera & 4B \\
\hline \multicolumn{6}{|l|}{ Sapotaceae } \\
\hline $\begin{array}{l}\text { Pouteria salicifolia (Spreng.) } \\
\text { Radlk. }\end{array}$ & Folha & Globoide $^{\mathrm{M}}$ & Verde & Cecidomyiidae (Diptera) & $4 C^{*}$ \\
\hline \multicolumn{6}{|l|}{ Solanaceae } \\
\hline Solanum ramulosum Sendtn. & Folha & Lenticular $^{\mathrm{M}}$ & Verde & Cecidomyiidae (Diptera) & $4 \mathrm{D}$ \\
\hline \multicolumn{6}{|l|}{ Styracaceae } \\
\hline Styrax leprosus Hook. \&Arn. & Folha & Globoide $^{\mathrm{M}}$ & Cinza & Acari & $4 \mathrm{E}$ \\
\hline Styrax leprosus Hook. \&Arn. & Ramo & Globoide $^{P}$ & Marrom & Cecidomyiidae (Diptera) & $4 \mathrm{~F}$ \\
\hline Styrax leprosus Hook. \&Arn. & Ramo & Globoide $^{\mathrm{P}}$ & Marrom & Hymenoptera & $4 \mathrm{G}$ \\
\hline \multicolumn{6}{|l|}{ Symplocaceae } \\
\hline $\begin{array}{l}\text { Symplocus uniflora (Pohl.) } \\
\text { Benth }\end{array}$ & Folha & Lenticular $^{\mathrm{M}}$ & Verde & Cecidomyiidae (Diptera) & $4 \mathrm{H}$ \\
\hline \multicolumn{6}{|l|}{ Verbenaceae } \\
\hline Lantana camara $\mathrm{L}$. & Folha & Globoide $^{\mathrm{M}}$ & Verde & $\begin{array}{l}\text { Schismatodiplosis lantanae (Rübsaamen, } \\
\text { 1908) (Diptera) }\end{array}$ & $4 \mathrm{I}$ \\
\hline $\begin{array}{l}\text { Lantana montevidensis } \\
\text { (Spreng.) Briq. }\end{array}$ & Ramo & Fusiforme $^{\mathrm{M}}$ & Marrom & Cecidomyiidae (Diptera) & $4 \mathrm{~J}$ \\
\hline \multicolumn{6}{|l|}{ Vitaceae } \\
\hline Cissus striata Ruiz \&Pav. & Ramo & Fusiforme $^{\mathrm{P}}$ & Vermelha & Cecidomyiidae (Diptera) & $4 \mathrm{~K}$ \\
\hline
\end{tabular}


FIGURA 1: Galhas induzidas por artrópodes em Floresta Estacional Semidecidual no Rio Grande do Sul, Brasil. Anacardiaceae [Lithraea brasiliensis (A), Schinnu spolygamus (B-C-D-E)], Asteraceae [Baccharis articulata (F), Baccharis dracunculifolia (G-H-I-J), Baccharis punctulata (K), Baccharis tridentata (L), Baccharis trimera (M-N), Dasyphyllum spinescens (O), Eupatorium inulifolium (P), Mikania glomerata (Q), Mikania hastato-cordata (R-S) Moquiniastrum polymorpha (T)].
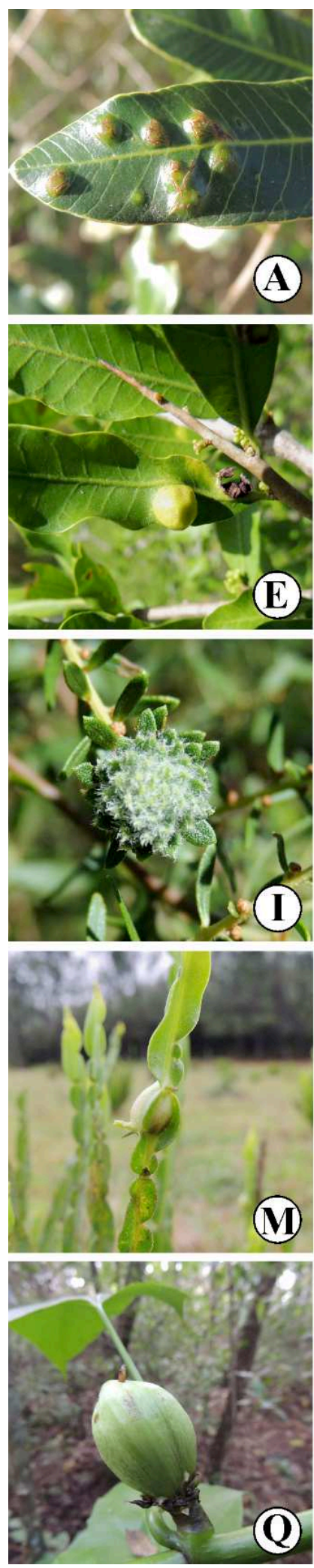
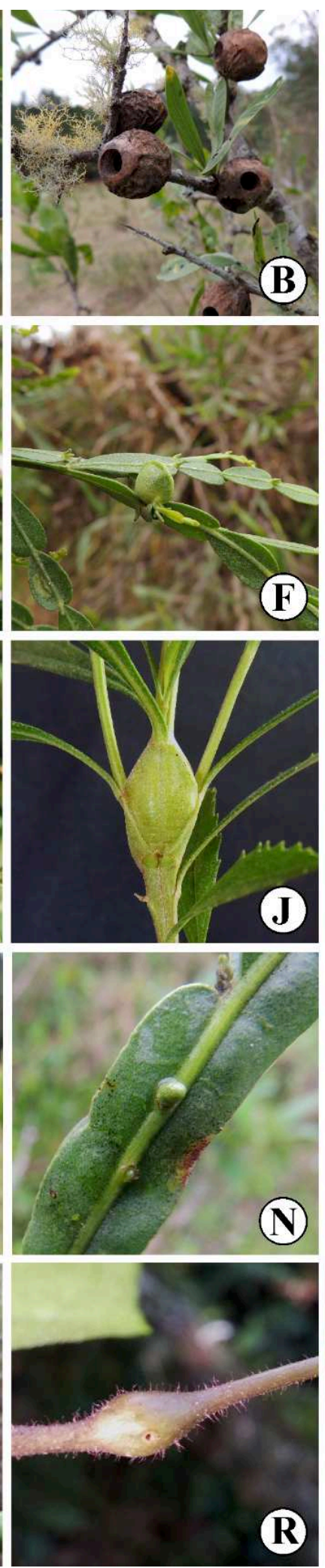
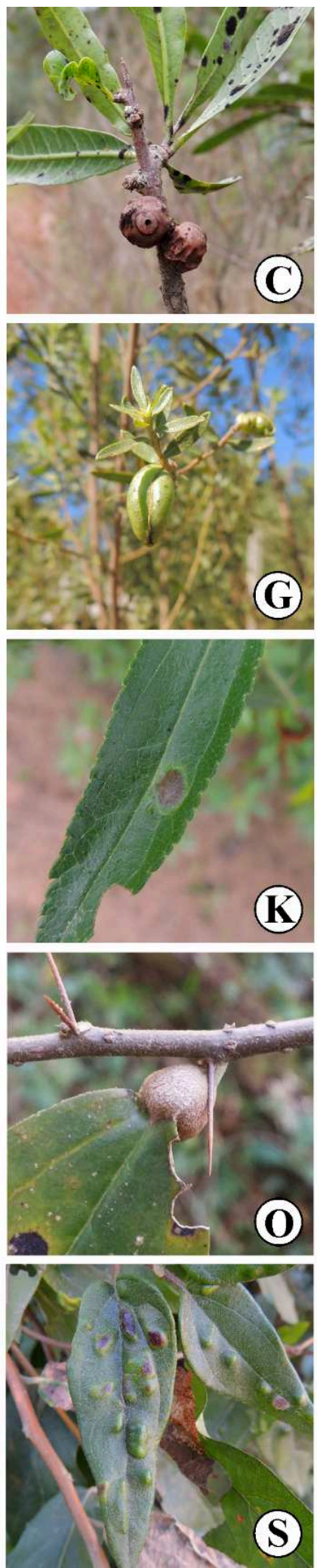
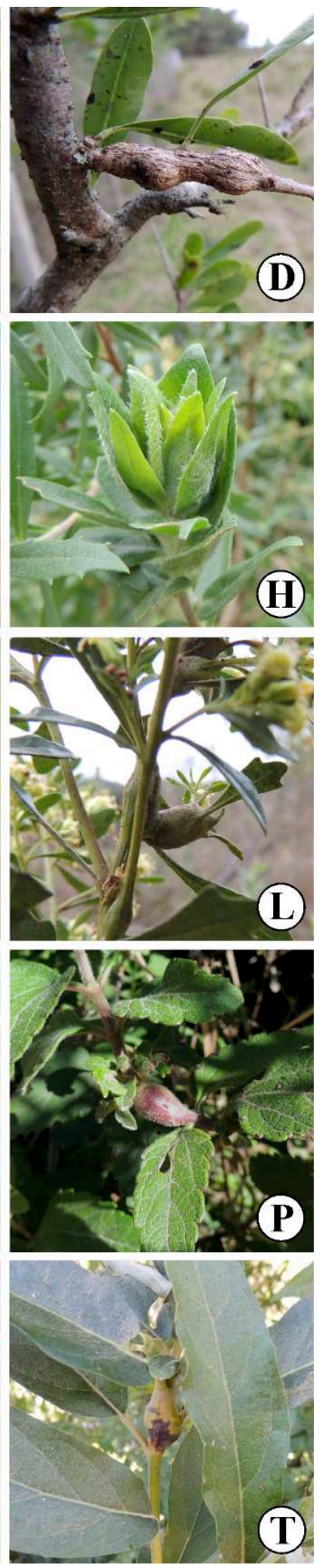
FIGURA 2: Galhas induzidas por artrópodes em Floresta Estacional Semidecidual no Rio Grande do Sul, Brasil. Asteraceae [Senecio brasiliensis (A), Solidago chilensis (B), Trixis praestans (C), Vernonanthura discolor (D)], Bignoniaceae [Pithecoctenium crucigerum (E)], Cannabaceae [Celtis iguanea (F)], Dioscoreaceae [Dioscorea multiflora (G)], Escalloniaceae [Escallonia bifida $(\mathrm{H})$ ], Euphorbiaceae [Sapium glandulosum (I), Sebastiania commersoniana (J-K-L-M)], Lamiaceae [Ocimun selloi $(\mathrm{N})$, Salvia guaranitica $(\mathrm{O})$ ], Lythraceae [Heimia myrtifolia (P)], Malvaceae [Luehea divaricata (Q-R-S), Sida sp. (T)].
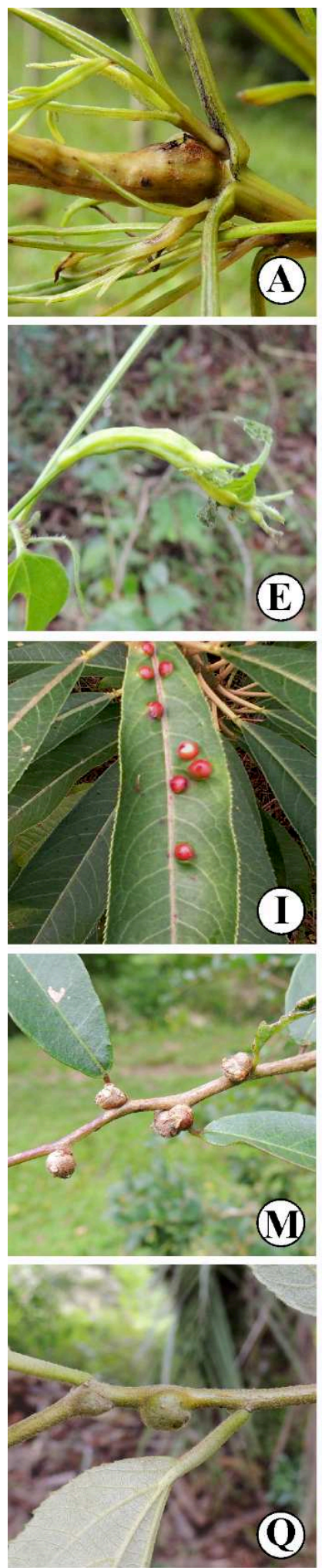
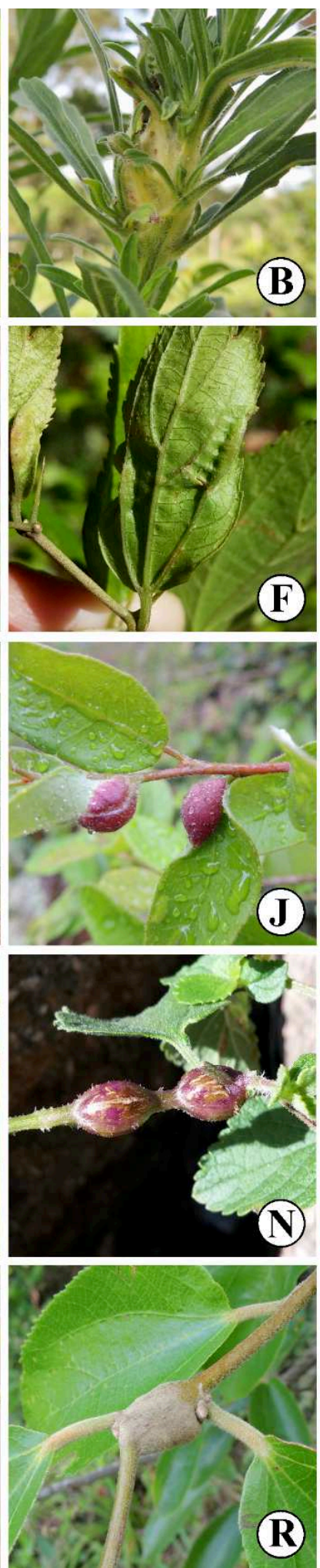
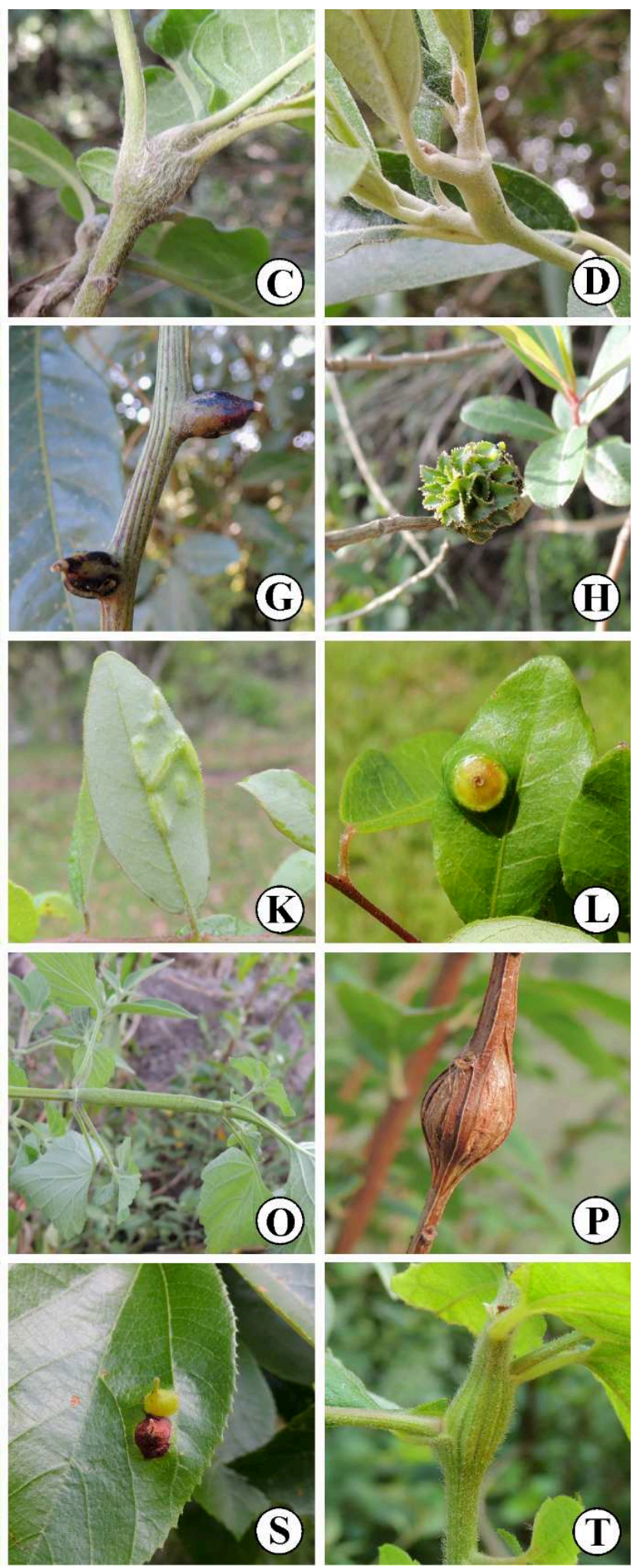
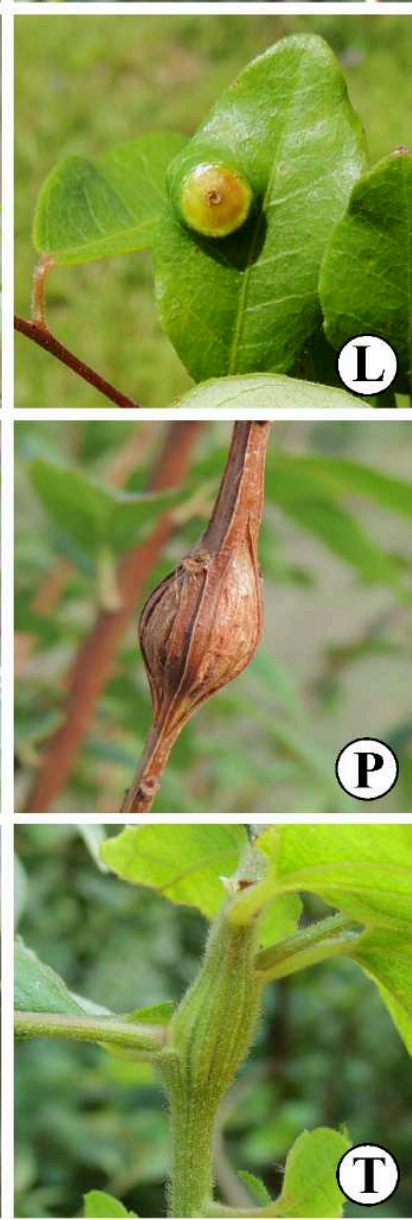
FIGURA 3: Galhas induzidas por artrópodes em Floresta Estacional Semidecidual no Rio Grande do Sul, Brasil. Melastomataceae [Miconia hiemalis (A), Miconia pusilliflora (B)], Meliaceae [Trichilia catigua (C)], Myrtaceae [Blepharocalyx salicifolius (D), Eugenia uniflora (E-F-G-H), Myrcianthes gigantea (I), Psidium cattleianum (J)], Nyctaginaceae [Guapira opposita (K-L)], Piperaceae [Piper xylosteoides $(\mathrm{M})]$, Polygonaceae[Polygonum acuminatum $(\mathrm{N})]$, Primulaceae $[$ Myrsine coriaceae $(\mathrm{O})$, Myrsine laetevirans (P), Myrsine umbellata (Q)], Rhamnaceae [Scutia buxifolia (R-S)], Rubiaceae [Psychotria carthagenensis (T)].
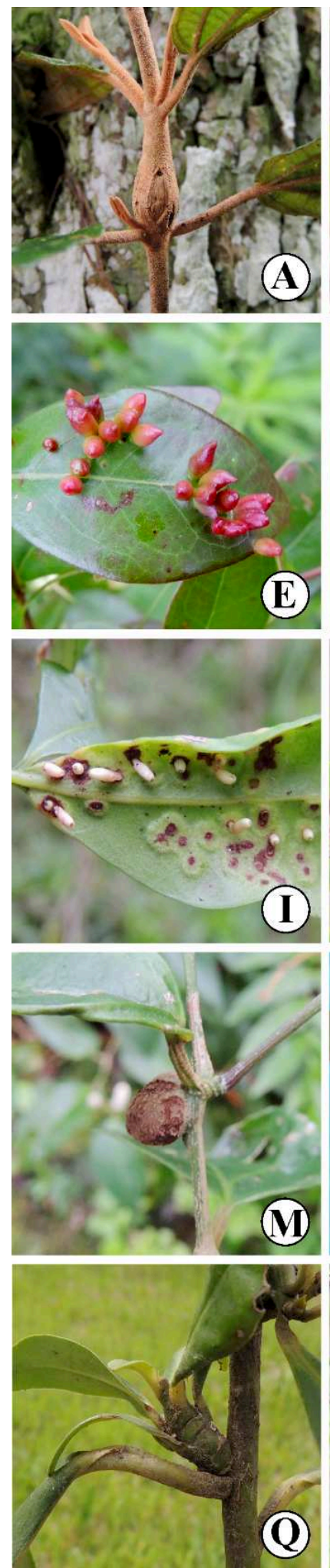
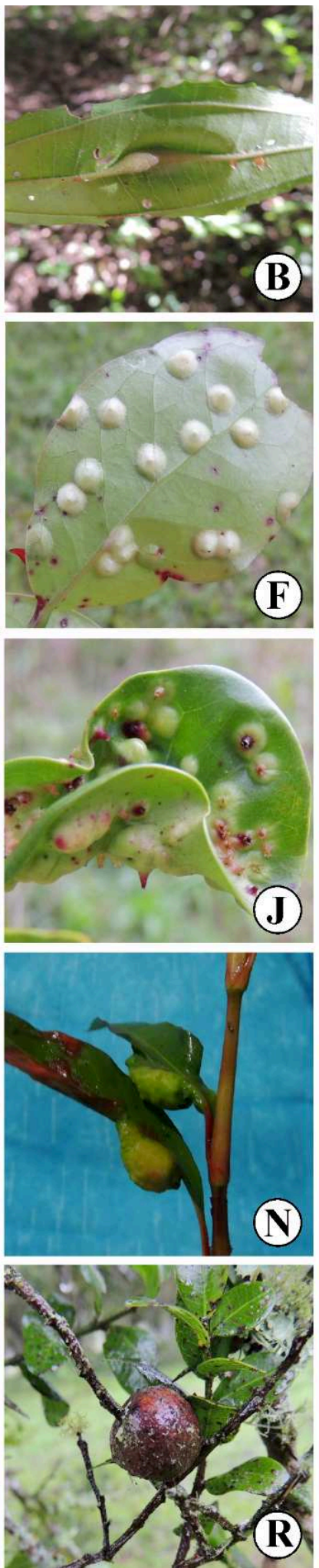
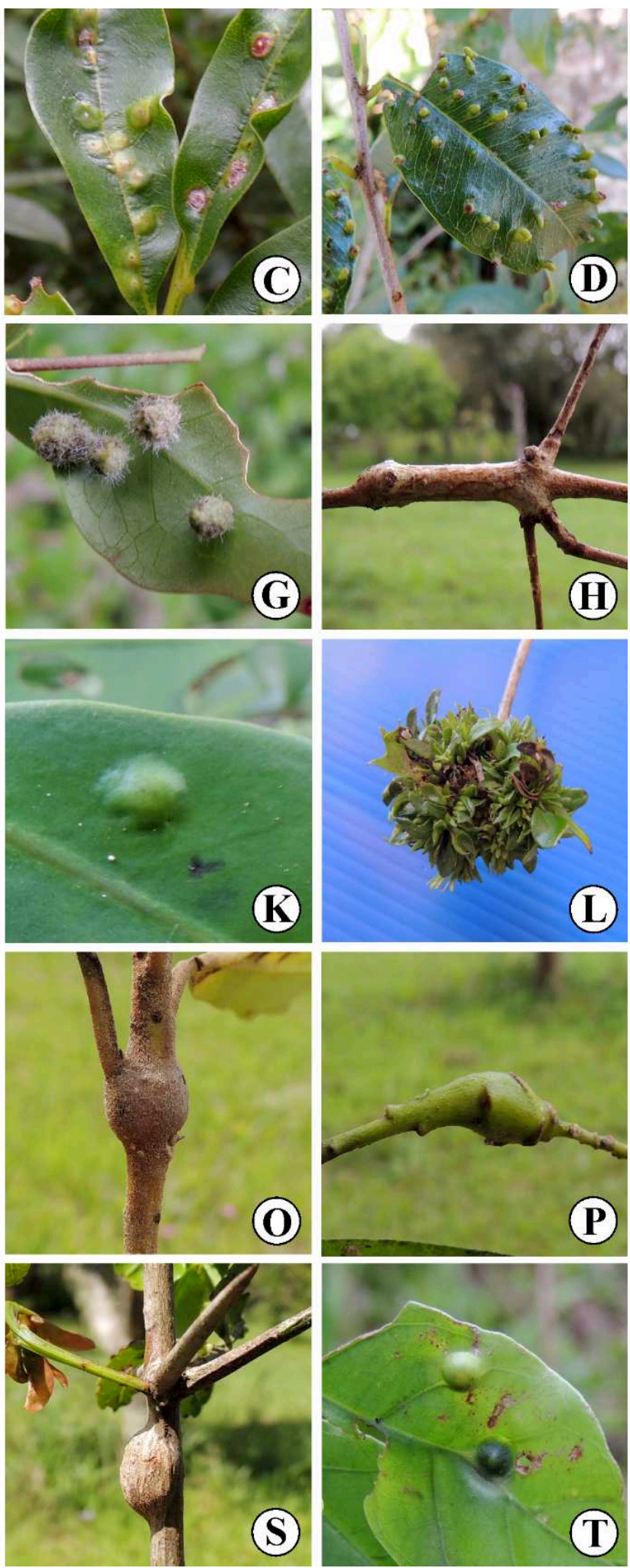
FIGURA 4: Galhas induzidas por artrópodes em Floresta Estacional Semidecidual no Rio Grande do Sul, Brasil. Salicaceae [Casearia sylvestris (A)], Sapindaceae [Allophylus edulis (B)], Sapotaceae [Pouteria salicifolia (C)], Solanaceae [Solanum ramulosum (D)], Styracaceae [Styrax leprosus (E-F-G)], Symplocaceae [Symplocus uniflora (H)], Verbenaceae [Lantana camara (I), Lantana montevidensis (J)], Vitaceae [Cissus striata $(\mathrm{K})]$.
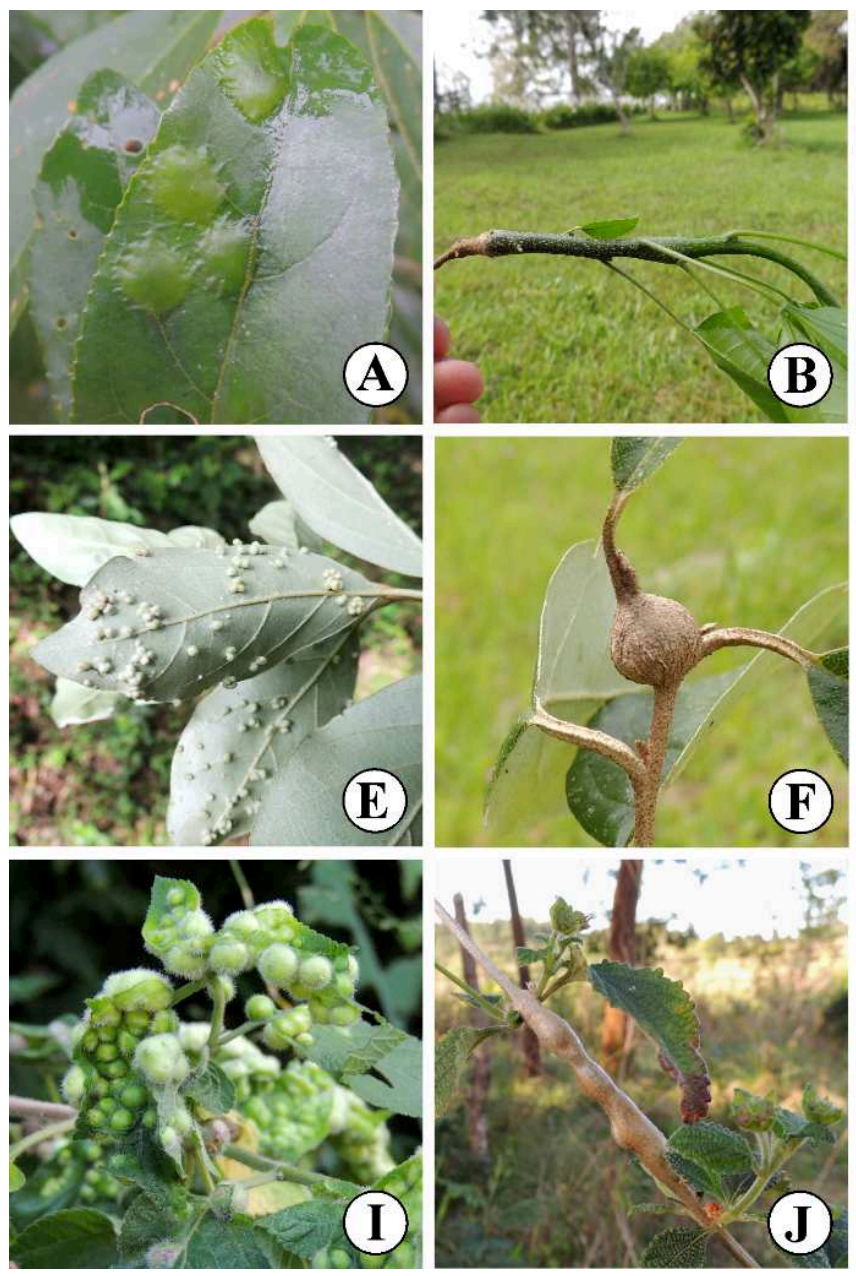
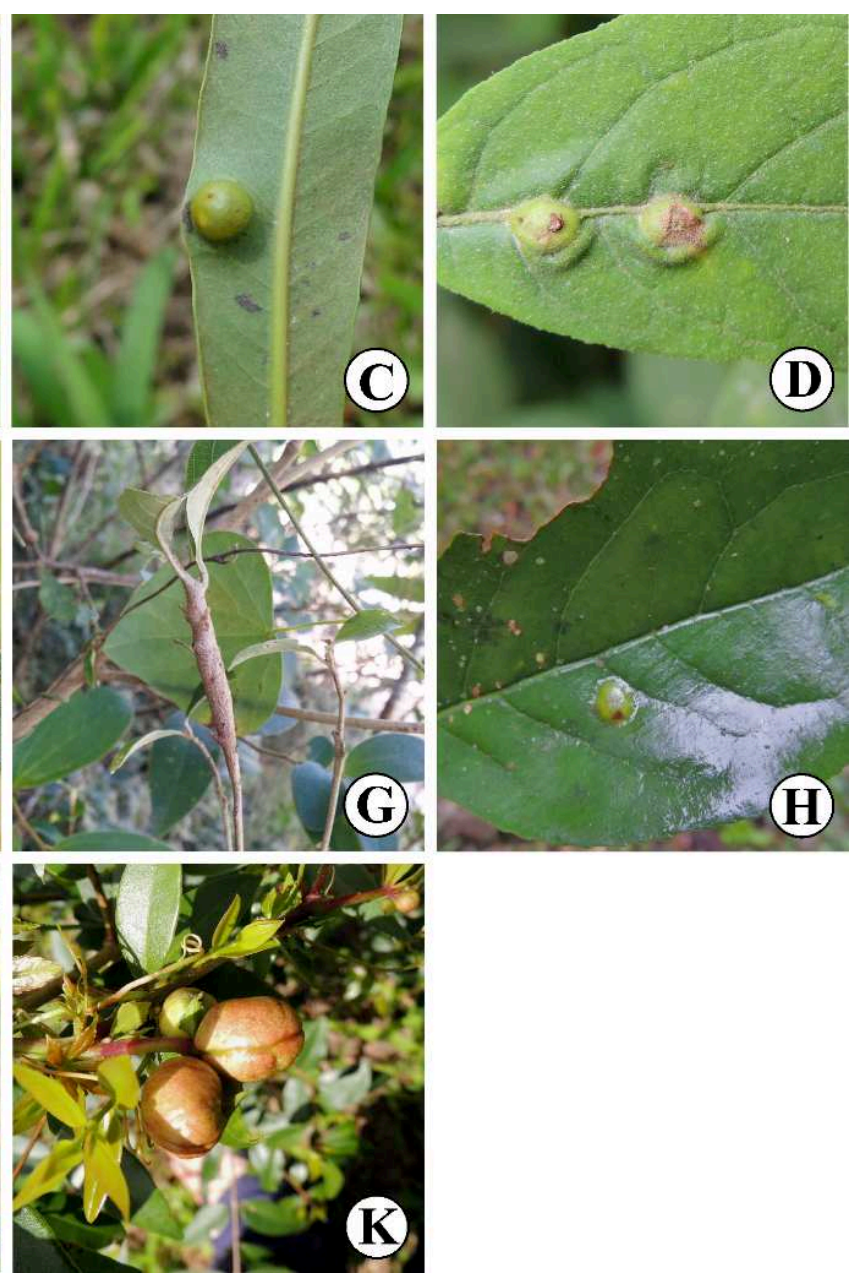

As galhas foram registradas em 51 espécies de plantas hospedeiras distribuídas em 42 gêneros e 27 famílias. As famílias que apresentaram maior riqueza de morfotipos de galhas foram Asteraceae $(n=19)$, seguida por Myrtaceae ( $\mathrm{n}=7)$, Anacardiaceae $(\mathrm{n}=$ $5)$ e Euphorbiaceae $(n=5)$. As quatro famílias juntas abrigam cerca de $50 \%$ do total de morfotipos, as demais registraram menos de quatro morfotipos.

O gênero de planta hospedeira com maior riqueza de galhas foi Baccharis L. ( $=9)$. Entre as espécies, Baccharis dracunculifolia DC (Asteraceae), Eugenia uniflora L. (Myrtaceae), Schinnus polygamus Cabrera
(Anacardiaceae) e Sebastiania commersoniana (Baill.) L.D.SM. \& Downs (Euphorbiaceae) registraram o maior número de morfotipos, quatro para cada uma das espécies hospedeiras.

A maioria das galhas foi induzida em ramos $(56 \%, \mathrm{n}=40)$ e folhas $(39 \%, \mathrm{n}=28)$, enquanto gemas representaram apenas $4 \%(n=3)$. A forma mais comum de galha foi fusiforme $(43,6 \%, \mathrm{n}=31)$, seguida por globoide $(24 \%, \mathrm{n}=17)$, lenticular $(18,3 \%, \mathrm{n}=13)$, roseta $(5,6 \%, n=4)$, amorfa $(2,8 \%, n=2)$, clavada $(2,8 \%, n=$ $2)$, bivalve $(1,4 \%, n=1)$ e cônica $(1,4 \%, n=1)$. 


\section{Discussão}

A riqueza de morfotipos de galhas registrada (71) foi superior à encontrada em inventários realizados em áreas de Floresta Estacional Semidecidual em outros locais do Brasil, como, por exemplo, na Floresta da Cicuta, Volta Redonda, Rio de Janeiro (FLOR et al., 2018; 43 morfotipos), no Parque da Sapucaia, Monte Carlos, Minas Gerais (COSTA et al., 2019; 29 morfotipos), em Altinópolis, São Paulo (RIBEIRO et al., 2019; 41 morfotipos) e em Telêmaco Borba, Paraná (SANTOS; RIBEIRO, 2015, 41 morfotipos).

Outro estudo, realizado no mesmo tipo de floresta também no Rio Grande do Sul, apresentou maior riqueza de espécies em relação aos nossos resultados (89 morfotipos) (GOETZ et al., 2018). Entretanto, os autores também coletaram em áreas da Floresta Estacional Decidual. Se excluirmos os registros dessas áreas, a riqueza registrada para a Floresta Estacional Semidecidual neste estudo foi igual a 57 morfotipos, inferior ao nosso resultado.

Ainda que sejam consideradas entre os estudos as diferenças de esforço amostral, riqueza de plantas hospedeiras e outras variáveis, nossos dados revelam o potencial desse tipo de floresta em relação à diversidade de galhadores. O registro de 18 novos morfotipos torna isso mais evidente e corrobora com trabalhos que preveem que a diversidade de galhadores é alta e pouco amostrada, como é o caso do Sul do Brasil (ARAÚJO, 2018).

Nossos resultados também reforçam a importância de Cecidomyiidae (Diptera) como o principal grupo de galhadores na região Neotropical (GAGNÉ; JASCHHOF, 2017) e biomas brasileiros (ARAÚJO et al., 2019), já que Cecidomyiidae foi o táxon dominante entre os indutores de galhas neste estudo. No Brasil, Asteraceae, Fabaceae e Myrtaceae são as famílias hospedeiras de artrópodes galhadores mais importantes (ARAÚJO et al., 2019). Frequentemente, Fabaceae é citada como a família com a maior riqueza de espécies em inventários realizados na Mata Atlântica (e.g. MAIA, 2013b; MAIA; CARVALHO-FERNANDES, 2016), entretanto, nossos resultados diferem dos encontrados nesses estudos. Além de registrarmos Asteraceae como a família com o maior número de morfotipos, não encontramos espécies de Fabaceae com galhas, apesar dessa família ser uma importante hospedeira no RS (MENDONÇA et al., 2014). Asteraceae também já havia sido citada em estudos anteriores no RS como a família com maior riqueza de espécies (MENDONÇA, 2007; TOMA; MENDONÇA 2013; MENDONÇA et al., 2014; GOETZ et al., 2018), o que parece ser uma peculiaridade do Rio Grande do Sul em relação às demais localidades brasileiras.

É importante destacar que a maior riqueza de morfotipos em Asteraceae neste estudo está associada à presença de espécies de Baccharis, já que nove dos 19 morfotipos registrados na família foram encontrados nas espécies do gênero. Espécies e gêneros de plantas podem ser considerados super hospedeiros quando apresentam muitos morfotipos de galhas (VELDTMAN; MCGEOCH, 2003). Esse é o caso de Baccharis, um dos gêneros mais ricos em espécies de galhadores na região Neotropical (FERNANDES et al., 1996) e também o segundo mais rico no RS (MENDONÇA et al., 2014). Entre as espécies registradas, B. dracunculifolia, E. uniflora, S. polygamus e $S$. commersoniana apresentaram o maior número de morfotipos. Todas essas espécies também já haviam sido citadas como super hospedeiras no RS (MENDONÇA et al., 2014).

A maior parte dos morfotipos foi registrada em ramos, um resultado incomum para inventários de galhadores em domínios da Mata Atlântica, que frequentemente relatam a predominância de galhas em folhas (e.g. MAIA et al., 2014; MAIA; CARVALHOFERNANDES, 2016; FLOR et al., 2018). Esse resultado incomum pode ter relação com a perda das folhas durante um período do ano neste tipo de floresta (Semidecidual), portanto, ramos representam um recurso constante para oviposição quando comparado às folhas (GOETZ et al., 2018).

Ramos também podem ser considerados sítios de oviposição mais seguros do que as folhas, pois oferecem maior proteção contra os herbívoros e a ação do vento (VELDTMAN; MCGEOCH, 2003). Contudo, inventários anteriores realizados no RS relatam proporções similares de morfotipos em ramos e folhas em áreas de Floresta Ombrófila Mista (TOMA; 
MENDONÇA, 2013) e de Florestas Estacionas Decidual e Semidecidual (GOETZ et al., 2018). Além disso, a predominância de galhas em ramos no RS já tinha sido citada em outro estudo mais amplo que considera diferentes tipos de vegetação e localidades (MENDONÇA et al., 2014). Essa característica parece ser outra peculiaridade do RS em relação a outros locais no Brasil. O formato fusiforme é comum em galhas de ramo (ISAIAS et al., 2013) e a sua predominância nesse trabalho pode estar associada à maior frequência de morfotipos neste órgão vegetal, sendo que a maior parte das galhas registrada nesse órgão apresentava esse formato.

Este estudo representa uma importante contribuição para o conhecimento dos artrópodes galhadores da Floresta Estacional Semidecidual no Sul do Brasil. Em alguns aspectos, as características das galhas seguiram tendências já relatadas para a região Neotropical. Entretanto, algumas particularidades se mostraram semelhantes a estudos também realizados no RS, provavelmente refletindo algum padrão biogeográfico que deve ser melhor investigado em estudos futuros. Além disso, poucos inventários de galhas incluem as galhas de Ácaros, diversidade que pouco conhecemos atualmente.

\section{Agradecimentos}

Aos colegas botânicos da UFRGS pela identificação das plantas. Ao CNPq e à CAPES pelas bolsas aos autores.

\section{Referências}

APG IV. An update of the Angiosperm Phylogeny Group classification for the orders and families of flowering plants: APG IV. Botanical Journal of the Linnean Society, London, v. 181, p. $1-20,2016$.

ARAÚJO, W. S. D. 30 years of research on insect galls in Brazil: a scientometric review. Papéis Avulsos de Zoologia, São Paulo, v. 58, e20185834, 2018.

ARAÚJO, W. S.; FERNANDES, G. W.; SANTOS, J. C. An overview of inventories of gall-inducing insects in Brazil: looking for patterns and identifying knowledge gaps. Anais da Academia Brasileira de Ciências, Rio de Janeiro, v. 91, n. 1, e20180162, 2019.
BREGONCI, J. D. M.; POLYCARPO, P. V.; MAIA, V. C. Galhas de insetos do Parque Estadual Paulo César Vinha (Guarapari, ES, Brasil). Biota Neotropica, Campinas, v. 10 n. 1, p. 265-274, 2010. CARNEIRO, M. A. A.; BORGES, R. A. X.; ARAÚJO, A. P. A.; FERNANDES, G.W. 2009. Insetos indutores de galhas da porção sul da Cadeia do Espinhaço, MG, Brasil. Revista Brasileira de Entomologia, Curitiba, v. 53, p. 570-592, 2009.

COSTA, K. C. D. S.; ARAÚJO, W. S. D. Distribution of gallinducing arthropods in areas of deciduous seasonal forest of Parque da Sapucaia (Montes Claros, MG, Brazil): effects of anthropization, vegetation structure and seasonality. Papéis Avulsos de Zoologia, São Paulo, v. 59, 2019.

FERNANDES, G. W.; CARNEIRO, M. A. A.; LARA, A. C. F.; ALLAIN, L. R.; ANDRADE, G. I.; JULIÃO, G. R.; REIS, T. R.; Silva, I. M. Galling insects on neotropical species of Baccharis (Asteraceae). Tropical Zoology, Firenze, v. 9, p. 315-332, 1996.

FLOR, I. C.; FLOR, J. C. R.; FURTADO, P. S. D. N. Insect galls of the Floresta da Cicuta (Volta Redonda, RJ, Brazil). Papéis Avulsos de Zoologia, São Paulo v. 58, e20185824, 2018.

GAGNÉ, R. J.; JASCHHOF, M. A Catalog of the Cecidomyiidae (Diptera) of the World. 4. ed. Digital. 2017. 762 p. Disponível em <https://www.ars.usda.gov/ARSUserFile s/80420580/ Gagne_2017_World_Cat_4th_ed.pdf.>.

GOETZ, A. P. M.; LUZ, F. A.; TOMA, T. S.; MENDONÇA JR, M. D. S. Gall-inducing insects of deciduous and semideciduous forests in Rio Grande do Sul State, Brazil. Iheringia. Série Zoologia, Porto Alegre, v. 108, e2018015, 2018.

GRANDEZ-RIOS, J. M.; GARCÍA-VILL ACORTA, R.; CUEVASREYES, P.; ARAÚJO W. S. Insectos inductores de agallas en América Latina: ecología, importancia y nuevas perspectivas. Revista de Biologia Neotropical, Goiânia, v. 12, n. 2, p. 92-103, 2015.

IBGE - INSTITUTO BRASILEIRO DE GEOGRAFIA E ESTATÍSTICA. Manual técnico da vegetação brasileira. Rio de Janeiro: IBGE, 2012. 271 p.

ISAIAS, R. M. S.; CARNEIRO, R. G. S.; OLIVEIRA, D. C.; SANTOS, J. C. Illustrated and annotated checklist of Brazilian gall morphotypes. Neotropical Entomology, Londrina, v. 42, p. 230 239, 2013.

MAIA, V. C. The gall midges (Diptera, Cecidomyiidae) from three restingas of Rio de Janeiro State, Brazil. Revista Brasileira de Zoologia, Curitiba, v. 18, n. 2, p. 583-629, 2001.

MAIA, V. C. Galhas de insetos em restingas da região sudeste do Brasil com novos registros. Biota Neotropica, Campinas, v. 13, n. 1, p. 183-209, 2013a.

MAIA, V. C. Insect galls of São Tomé das Letras (MG, Brazil). Biota Neotropica, Campinas, v. 13, n. 4, p. 164-189, 2013 b.

MAIA, V. C.; CARDOSO, L. J. T.; BRAGA, M. A. Insect galls from Atlantic Forest areas of Santa Teresa, Espírito Santo, Brazil: characterization and occurrence. Boletim do Museu de Biologia Mello Leitão. Nova Série, Santa Tereza, v. 33, p. 47-129, 2014.

MAIA, V. C.; CARVALHO-FERNANDES, S. P. Insect galls of a protected remnant of the Atlantic Forest tableland from Rio de Janeiro State (Brazil). Revista Brasileira de Entomologia, Curitiba, v. 60, n. 1, 40-56. 2016. 
MAIA, V. C.; MAGENTA, M. A.; MARTINS, S. E. Ocorrência e caracterização de galhas de insetos em áreas de restinga de Bertioga (São Paulo, Brasil). Biota Neotropica, Campinas, v. 8, n. 1, p. 167-197, 2008.

MAIA, V. C.; MASCARENHAS, B. Insect galls of the Parque Nacional do Itatiaia (Southeast region, Brazil). Anais da Academia Brasileira de Ciências, Rio de Janeiro, v. 89, n. 1, p. 505-575, 2017.

MAIA, V. C.; OLIVEIRA, J. C. Insect galls of the Reserva Biológica Estadual da Praia do Sul (Ilha Grande, Angra dos Reis, RJ). Biota Neotropica, Campinas, v. 10, n. 4, p. 227-238, 2010.

MAIA, V. C.; SIQUEIRA, E. D. S. Insect galls of the Reserva Biológica União, Rio de Janeiro, Brazil. Biota Neotropica, Campinas, v. 20, n. 1, e20190758, 2020.

MANI, M. S. Ecology of plant galls. Junk: The Hague, 1964. 434 p.

MOREnO, J. A. Clima do Rio Grande do Sul. Porto Alegre: Secretaria da Agricultura, 1961. 42 p.

MENDONÇA, M. S. Plant diversity and galling arthropod diversity searching for taxonomic patterns in an animal-plant interaction in the neotropics. Boletín de la Sociedad Argentina de Botánica, Córdoba, v. 42, p. 347-357, 2007.

MENDONÇA, M. de S.; TOMA, T. S. P.; SILVA, J. S. Galls and galling arthropods of Southern Brazil. In: FERNANDES, G. W.; SANTOS, J. C. (Ed.). Neotropical insect galls. New York: Springer, 2014. p. 221-256.

RAMAN, A. Insect-induced plant galls of India: unresolved questions. Current Science, Bangalore, v. 92, n. 6, p. 748-757, 2007.
RIBEIRO, A. D. N.; BALBI, M. I. P. D. A.; URSO-GUIMARÃES, M. V. Characterization of insect galls from a vegetation area in Altinópolis, São Paulo State, Brazil. Papéis Avulsos de Zoologia, São Paulo, v. 59, e20195904, 2019.

SANTOS, P. O.; RIBEIRO, J. E. L. S. Ocorrência e caracterização de galhas em fragmento de Floresta Estacional Semidecidual em Telêmaco Borba, Paraná, Brasil. Semina, Ciências Biológicas e da Saúde, Londrina, v. 36, n. 2, p. 15-24, 2015.

SHORTHOUSE, J. D.; WOOL, D.; RAMAN, A. Gall-inducing insects - Nature's most sophisticated herbivores. Basic and Applied Ecology, Berlin, v. 6, n. 5, p. 407-411, 2005.

STONE, G. N.; SCHÖNROGGE. K. The adaptive significance of insect gall morphology. Trends in Ecology \& Evolution, London, v. 18, p. 512-522, 2003.

TEIXEIRA, M. B.; COURA NETO, A. B.; PASTORE, U.; RANGEL FILHO, A. L. R. Vegetação. As regiões fitoecológicas, sua natureza e seus recursos econômicos. Estudo fitogeográfico. Levantamento de recursos naturais. Instituto Brasileiro de Geografia e Estatística, Rio de Janeiro, v. 33, p. 541-632, 1986.

TOMA, T. S. P.; MENDONÇA, M. S. Gall-inducing insects of an Araucaria Forest in southern Brazil. Revista Brasileira de Entomologia, Curitiba, v. 57, p. 225-233, 2013.

VELDTMAN, R.; MCGEOCH, M. A. Gall forming insect species richness along a non-scleromorphic vegetation rainfall gradient in South Africa: the importance of plant community composition. Austral Ecology, Alice Springs, v. 28, p. 1-13, 2003. 